\title{
Enfuvirtide, an HIV-1 Fusion Inhibitor, for Drug-Resistant HIV Infection in North and South America
}

\author{
Jacob P. Lalezari, M.D., Keith Henry, M.D., Mary O’Hearn, M.D., Julio S.G. Montaner, M.D., Peter J. Piliero, M.D., \\ Benôit Trottier, M.D., Sharon Walmsley, M.D., Calvin Cohen, M.D., Daniel R. Kuritzkes, M.D., Joseph J. Eron, Jr., M.D., \\ Jain Chung, Ph.D., Ralph DeMasi, Ph.D., Lucille Donatacci, M.S., Claude Drobnes, M.D., John Delehanty, Ph.D., \\ and Miklos Salgo, M.D., Ph.D., for the TORO I Study Group*
}

\section{ABSTRACT}

BACKGROUND

The T-20 vs. Optimized Regimen Only Study 1 (TORO 1) was a randomized, open-label, phase 3 study of enfuvirtide (T-20), a human immunodeficiency virus type 1 (HIV-1) fusion inhibitor.

METHODS

Patients from 48 sites in the United States, Canada, Mexico, and Brazil with at least six months of previous treatment with agents in three classes of antiretroviral drugs, resistance to drugs in these classes, or both, and with at least 5000 copies of HIV-1 RNA per milliliter of plasma were randomly assigned in a 2:1 ratio to receive enfuvirtide plus an optimized background regimen of three to five antiretroviral drugs or such a regimen alone (control group). The primary efficacy end point was the change in the plasma HIV-1 RNA level from base line to week 24 .

RESULTS

A total of 501 patients underwent randomization, and 491 received at least one dose of study drug and had at least one measurement of plasma HIV-1 RNA after treatment began. The two groups were balanced in terms of the median base-line HIV-1 RNA level (5.2 $\log _{10}$ copies per milliliter in both groups), median CD4+ cell count (75.5 cells per cubic millimeter in the enfuvirtide group, and 87.0 cells per cubic millimeter in the control group), demographic characteristics, and previous antiretroviral therapy. At 24 weeks, the least-squares mean change from base line in the viral load (intention-to-treat, last observation carried forward) was a decrease of $1.696 \log _{10}$ copies per milliliter in the enfuvirtide group, and a decrease of $0.764 \log _{10}$ copies per milliliter in the control group ( $\mathrm{P}<0.001)$. The mean increases in CD4+ cell count were 76 cells per cubic millimeter and 32 cells per cubic millimeter, respectively $(\mathrm{P}<0.001)$. Reactions at the site of the injections were reported by 98 percent of patients receiving enfuvirtide. There were more cases of pneumonia in the enfuvirtide group than in the control group.

CONCLUSIONS

The addition of enfuvirtide to an optimized antiretroviral regimen provided significant antiretroviral and immunologic benefit through 24 weeks in patients who had previously received multiple antiretroviral drugs and had multidrug-resistant HIV-1 infection.
From Quest Clinical Research, Mount Zion Hospital, and the University of California, San Francisco, San Francisco (J.P.L.); the HIV Program, Hennepin County Medical Center, Minneapolis (K.H.); the Oregon Health and Science University, Portland (M.O.); St. Paul's Hospital and the University of British Columbia, Vancouver, Canada (J.S.G.M.); the Clinical Research Initiative, Albany Medical College, Albany, N.Y. (P.J.P.); the Clinique Médicale l'Actuel, Montreal (B.T.); the University of Toronto, Toronto (S.W.); the Community Research Initiative of New England, Boston (C.C.); the Division of Infectious Diseases, University of Colorado Health Sciences Center, Denver (D.R.K.); the University of North Carolina at Chapel Hill School of Medicine, Chapel Hill (J.J.E.); Roche, Nutley, N.J. (J.C., L.D., M.S.); and Trimeris, Durham, N.C. (R.D., C.D., J.D.). Address reprint requests to $D r$. Lalezari at Quest Clinical Research, 2300 Sutter St., Suite 202, San Francisco, CA 94115, or at drjay@questclinical.com.

*Members of the TORO 1 (T-20 vs. Optimized Regimen Only Study 1) group are listed in the Appendix.

This article was published at www.nejm.org on March 13, 2003.

N EnglJ Med 2003;348:2175-85. 
T

HE DURABILITY OF SUPPRESSION OF human immunodeficiency virus (HIV) infection with antiretroviral therapy is often limited, for reasons that include poor penetration into protected sites containing a reservoir of $\mathrm{HIV},{ }^{1,2}$ drug toxicity, ${ }^{3,4}$ alterations in the bioavailability and metabolism of antiretroviral drugs (e.g., interactions between drugs), ${ }^{5}$ and lack of adherence to complex treatment regimens. ${ }^{6,7}$ These factors contribute to persistent viral replication in patients receiving therapy, increasing the risk of viral resistance, which can limit future treatment options. ${ }^{8,9}$ Salvage therapy after viral rebound is more successful if an agent from a class of antiretroviral drugs to which the patient has not previously been exposed is included in the regimen.

Enfuvirtide (also known as T-20) is a novel, synthetic, 36-amino-acid peptide that binds to a region of the envelope glycoprotein 41 of HIV type 1 (HIV-1) that is involved in the fusion of the virus with the membrane of the CD4+ host cell. ${ }^{10}$ This agent exhibits potent and selective inhibition of HIV-1 in vitro without cytotoxicity ${ }^{10}$ and is the first inhibitor of HIV entry to show consistent potent activity in persons infected with HIV-1. ${ }^{11-14}$

In the T-20 vs. Optimized Regimen Only Study 1 (TORO 1), we compared the effect of enfuvirtide in combination with an antiretroviral regimen that was optimized with the aid of phenotypic and genotypic resistance testing with the effect of an optimized regimen alone on plasma HIV-1 RNA levels and CD4+ cell counts in patients who had previously received multiple antiretroviral drugs and carried virus that was resistant to all three currently available classes of antiretroviral drugs. A similar study (T-20 vs. Optimized Regimen Only Study 2 [TORO 2]) was conducted in Europe and Australia. ${ }^{15}$ In this article, we present the results of the week-24 primary efficacy and safety analyses of TORO 1.

METHODS

\section{STUDY DESIGN}

We conducted a randomized, open-label, multicenter, phase 3 study comprising a 6 -week screening phase followed by 48 weeks of treatment, with an optional 48-week extension of treatment, and 4 weeks of follow-up for safety analyses. An initial screening visit, occurring three to six weeks before randomization, included a complete medical history taking, measurement of plasma HIV-1 RNA (Amplicor HIV-1 Monitor, version 1.5, Roche), and genotypic and phenotypic resistance testing (per- formed by ViroLogic, San Francisco). A second measurement of plasma HIV-1 RNA and safety assessments were performed at a second screening visit one to two weeks before randomization. An optimized regimen of three to five antiretroviral drugs was selected by the investigator and the patient before randomization on the basis of the patient's previous antiretroviral treatment and tolerance history and the results of previous and screening genotypic and phenotypic resistance testing.

Qualifying patients underwent randomization according to a centralized, adaptive randomization scheme and were assigned in a 2:1 ratio to one of two groups: enfuvirtide plus the optimized background regimen (enfuvirtide group) or the optimized regimen alone (control group). Randomization was stratified according to the second plasma HIV-1 RNA measurement ( $<40,000$ or $\geq 40,000$ copies per milliliter) and according to the use or nonuse of newly approved or investigational antiretroviral drugs (lopinavir-ritonavir, tenofovir, or both) in the optimized background regimen.

Virologic failure was defined by either a decrease from base line of less than $0.5 \log _{10}$ copies per milliliter in plasma HIV-1 RNA on two or three consecutive measurements after week 6 , with at least 14 days between the first and last measurements, a decrease from base line of less than $1.0 \log _{10}$ copies per milliliter in plasma HIV-1 RNA on consecutive measurements (as above) after week 14, or a decrease from base line of at least $2.0 \log _{10}$ copies per milliliter in plasma HIV-1 RNA on consecutive measurements (as above) followed by a rebound of more than $1.0 \log _{10}$ copies per milliliter in plasma HIV-1 RNA from the average of the two lowest values (not necessarily consecutive) after week 6 . All patients in whom the criteria for virologic failure were met after week 8 underwent repeated genotypic and phenotypic resistance testing and were encouraged to change their background regimen. In these cases, a background regimen of more than five drugs was permitted. Patients in the enfuvirtide group could continue to receive enfuvirtide, and patients in the control group were permitted to add enfuvirtide to their revised regimen. Patients with virologic failure who did not want to switch to or to continue to receive enfuvirtide were allowed to remain in the study for a maximum of one month.

The results on the screening resistance tests were used to define phenotypic and genotypic sensitivity scores. The genotypic sensitivity score was the total number of drugs in the optimized background regimen to which a patient's viral isolate showed geno- 
typic sensitivity according to a modification of a previously published interpretation algorithm. ${ }^{16}$ For tenofovir, genotypic resistance was defined as the presence of $\mathrm{K} 65 \mathrm{R}$, or three or more of the thymidine analogue-associated resistance mutations (M41L, D67N, K70R, L210W, T215Y, T215F, K219Q, K219E, or K219N), including either M41L or L210W. The phenotypic sensitivity score was the total number of drugs in the optimized background regimen to which a patient's viral isolate showed phenotypic sensitivity. For tenofovir, the phenotypic sensitivity was set equal to the genotypic sensitivity.

\section{STUDY POPULATION}

Patients were older than 16 years of age and had at least six months of previous treatment with at least one nucleoside reverse-transcriptase inhibitor, at least one nonnucleoside reverse-transcriptase inhibitor, and at least two protease inhibitors, documented resistance to these drugs, or both. Patients were eligible for the study if they had been receiving stable combination therapy or no antiretroviral therapy for four or more weeks before inclusion and had a plasma HIV-1 RNA level of at least 5000 copies per milliliter at both screening visits. Patients were ineligible for inclusion if they had already received treatment with enfuvirtide, the experimental fusion inhibitor T-1249, or both. Female patients were excluded if they were pregnant, breast-feeding, or planning to become pregnant during the study.

\section{CONSENT, APPROVALS, AND CONDUCT OF THE STUDY}

Written informed consent was obtained from all patients. Before the study began, the protocol and the informed-consent provisions were reviewed and approved by the independent ethics committee or institutional review board at each of the centers involved in the study.

Design of the trial protocol was the responsibility of the study sponsors in collaboration with various health authorities and advisory boards, which included some coauthors of the article. All statistical analyses were executed by employees of the study sponsors. Data were collected by the study sponsors. Interpretation of the data was performed by the study sponsors in collaboration with the advisory boards and the clinical investigators.

\section{STUDY MEDICATION}

Enfuvirtide $(90 \mathrm{mg})$ was administered twice daily by subcutaneous injection into the abdomen, the upper arm, or the anterior aspect of the thigh. The first injection was administered by study personnel at the study site, and patients were trained in sterile technique and instructed in reconstituting and injecting enfuvirtide.

The optimized background regimen could include tenofovir, lopinavir-ritonavir, or both. Both of these agents were investigational at the start of the study but were approved in most countries during the course of the study. Ritonavir at doses of $266 \mathrm{mg}$ per day or less (i.e., booster doses) was not counted as an active component in either a prestudy regimen or the background regimen. Changes in the background regimen were permitted only in the event of protocol-defined virologic failure or management of toxic effects. Adherence in both treatment groups was calculated with the use of a patient questionnaire that assessed the number of missed doses of enfuvirtide or oral antiretroviral drugs during the four days preceding each study visit.

\section{EFFICACY ANALYSIS}

The primary efficacy end point was the change from base line to 24 weeks in the plasma HIV-1 RNA level (measured on a logarithmic [base 10] scale). Secondary efficacy end points included the category of virologic response, the time to virologic failure, and the changes from base line to week 24 in the CD4+ cell count. Virologic response at week 24 was classified into three categories: an HIV-1 RNA level of less than 50 copies per milliliter, an HIV-1 RNA level of less than 400 copies per milliliter, or a decrease from base line in the HIV-1 RNA level of at least $1.0 \log _{10}$ copies per milliliter; two consecutive measurements were required for categorization.

\section{SAFETY ANALYSIS}

Safety end points included adverse events, serious adverse events (including death), adverse events leading to premature withdrawal from the study, local reactions at the site of the injections, and abnormal results on clinical laboratory tests (hematology, serum chemistry, and urinalysis). The severity of adverse events was graded according to the modified grading scale of the AIDS Clinical Trials Group. ${ }^{17}$ Causality was assessed in all cases; if the study treatment was deemed to have caused the event, investigators attributed it to the entire regimen, except in the case of serious adverse events, which were attributed to individual agents.

Injection-site reactions were assessed according to an overall grade that was based on the level of pain and discomfort. In the event of a grade 4 injection-site reaction (severe pain that was clinically sig- 
nificant or life-threatening or resulted in a new or prolonged hospitalization or persistent or substantial incapacity or death) or recurrent grade 3 injection-site reactions (severe pain requiring nontopical analgesic agents or limiting the patient's ability to engage in usual activities), enfuvirtide therapy was discontinued. All grade 4 injection-site reactions and laboratory abnormalities except grade 4 triglyceride values were also defined as serious adverse events.

An additional updated safety analysis combined data from the two phase 3 studies (TORO 1 and TORO 2). This combination offered a larger population on which to base a characterization of the safety profile of enfuvirtide and was appropriate because the studies have similar designs, criteria for patient selection, and protocol-specified analyses. For this update, a separate analysis investigated the incidence of combinations of adverse events that might be considered clinically equivalent in order to identify whether small increases in the incidence of several adverse events would, when combined, show a relevant difference between treatment groups.

\section{STATISTICAL ANALYSIS}

Data on efficacy were analyzed for an intentionto-treat population, defined as all patients who underwent randomization, received at least one dose of study medication, and had at least one plasma HIV-1 RNA measurement after treatment began. HIV-1 RNA values obtained after confirmed virologic failure were excluded from the analysis. Missing values were imputed for the analysis with the use of a last-observation-carried-forward method. The $\log _{10}$ HIV-1 RNA value at week 24 was defined as the mean of the last two $\log _{10}$ HIV-1 RNA values on completion of 24 weeks in the study, the mean of the two HIV-1 RNA values that confirmed virologic failure, or for patients who withdrew from the study, the mean of the last two $\log _{10}$ HIV-1 RNA values before withdrawal. Changes from base line to week 24 in $\log _{10}$ HIV-1 RNA levels and CD4+ cell counts were evaluated by analysis of covariance including terms for the randomization stratum (of plasma HIV-1 RNA level and use or nonuse of newly approved or investigational agents), the treatment group, and the interaction between stratum and treatment group, with the base-line phenotypic sensitivity score (for the evaluation of the change in the HIV-1 RNA level) or the base-line CD4+ cell count (for the evaluation of the change in the $\mathrm{CD} 4+$ cell count) as covariates.
To test the robustness of the results of the primary efficacy analysis based on the last-observation-carried-forward method of imputation, three sensitivity analyses were performed as follows: the change from base line in viral load was set at zero for patients who withdrew before week 24; the change from base line in viral load was set at zero for patients who withdrew or who had virologic failure before week 24; and in a cohort analysis, separate analyses were performed without the use of the last-observation-carried-forward method for patients who completed 4, 8, 12,16, 20, and 24 weeks of treatment.

For analysis of categories of virologic response (with patients who had missing data or virologic failure treated as having had no response), a stratified Mantel-Haenszel test was used. Time to virologic failure was estimated by the Kaplan-Meier method. A stratified log-rank test was used to compare the time-to-event curves of the two treatment groups.

\section{RESULTS}

\section{STUDY POPULATION}

A total of 501 patients underwent randomization at 48 centers in the United States, Canada, Mexico, and Brazil between December 2000 and June 2001. Of these patients, 491 (326 in the enfuvirtide group and 165 in the control group) used the study medication at least once, had a follow-up visit to record safetyrelated data, and had an assessment of the HIV-1 RNA level after treatment began (Fig. 1).

\section{DEMOGRAPHIC AND BASE-LINE CHARACTERISTICS OF THE PATIENTS}

Demographic and base-line characteristics were similar in the two groups (Table 1). The genotypic sensitivity scores at base line (mean, 1.9 in each group) and the phenotypic sensitivity scores at base line (mean, 1.7 in the enfuvirtide group and 1.8 in the control group) indicated that HIV-1 from the majority of patients in each group was sensitive to less than two of the drugs used in the background regimen. Previous treatment with at least five protease inhibitors was reported for a slightly higher percentage of patients in the enfuvirtide group (49.4 percent) than in the control group (39.4 percent, $\mathrm{P}=0.04)$. The percentage of patients who had previously received lopinavir-ritonavir, classified as a newly approved or investigational antiretroviral drug in this study, was also higher in the enfuvirtide 


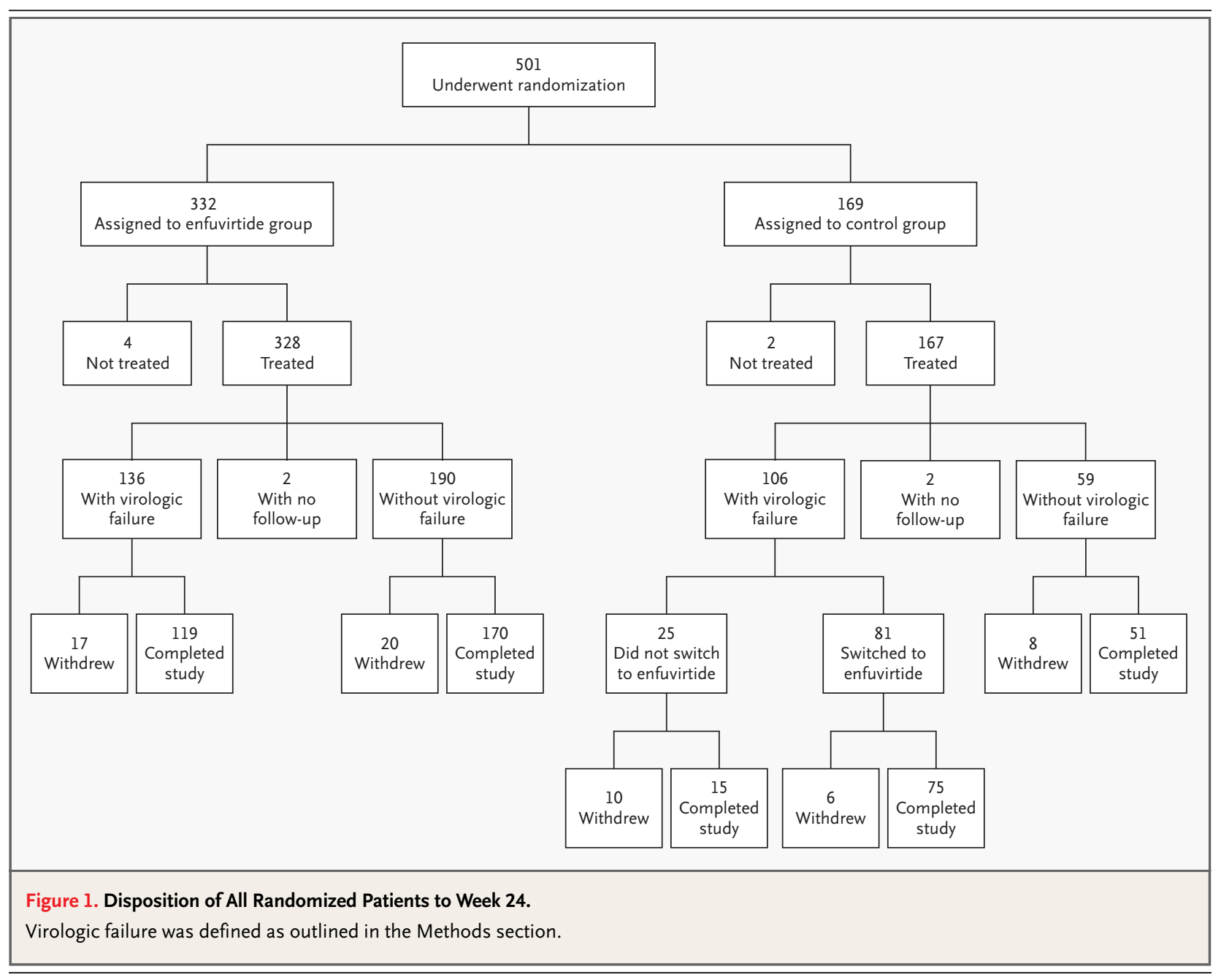

group (38.7 percent) than in the control group (27.9 percent, $\mathrm{P}=0.02$ ). In both groups, there was a mean of four drugs in the background regimen (Table 1).

\section{CHANGES IN TREATMENT AND PREMATURE WITHDRAWAL}

In the control group, 106 patients (64.2 percent) had protocol-defined virologic failure by week 24 , and 81 of these patients switched to enfuvirtide (Fig. 1). By week 24, 37 patients in the enfuvirtide group (11.3 percent), 18 patients remaining in the control group (21.4 percent), and 6 of the patients who had switched to enfuvirtide (7.4 percent) had withdrawn from the study.

\section{ADHERENCE}

The mean level of adherence to the enfuvirtide component of the regimen over the 24-week period was at least 85 percent in 92.9 percent of patients. The overall adherence to the entire regimen in the enfuvirtide group was at least 85 percent in 88.0 percent of patients. The mean level of adherence to the background regimen in the control group was at least 85 percent in 90.3 percent of patients.

\section{EFFICACY}

Changes in the Plasma HIV-1 RNA Level

The least-squares mean change from base line in the plasma HIV-1 RNA level was a decrease of 0.764 $\log _{10}$ copies per milliliter in the control group and a decrease of $1.696 \log _{10}$ copies per milliliter in the enfuvirtide group, representing a difference between groups of $0.933 \log _{10}$ copies per milliliter $(\mathrm{P}<$ 0.001) (Table 2). The least-squares mean differences significantly favored the enfuvirtide group in all four randomization strata $(\mathrm{P}<0.05$ for all comparisons). 


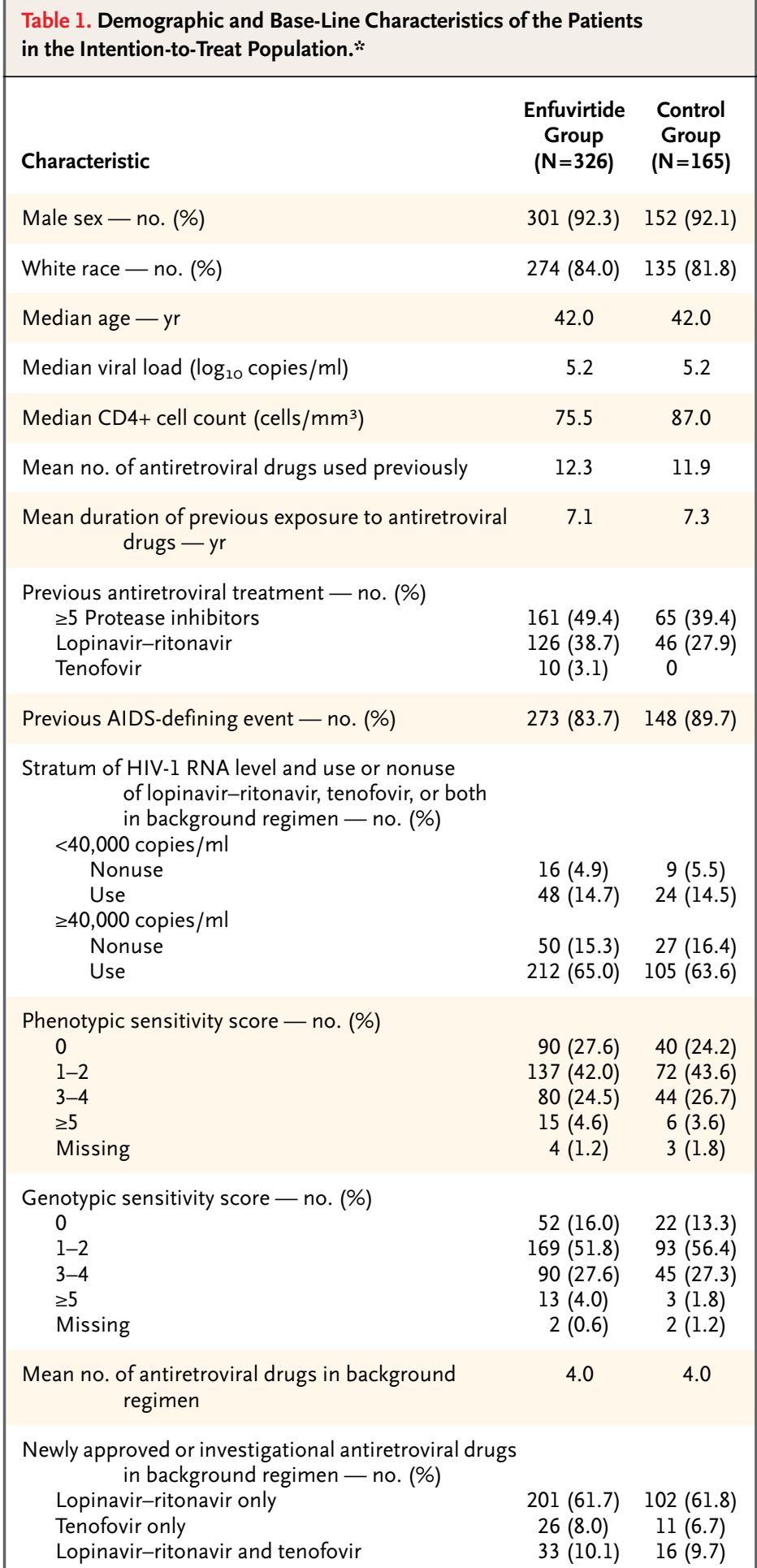

* There were 401 patients in the intention-to-treat population in the United States, 66 in Canada, 15 in Mexico, and 9 in Brazil. The genotypic and phenotypic sensitivity scores were calculated as described in the Methods section. AIDS denotes the acquired immunodeficiency syndrome.
The sensitivity analyses yielded least-squares mean differences of $0.888 \log _{10}$ copies per milliliter $(\mathrm{P}<$ 0.001 ) when the change from base line in viral load was set at zero for patients who withdrew from the study and $0.802 \log _{10}$ copies per milliliter $(\mathrm{P}<0.001)$ when the change from base line in viral load was set at zero for both patients who withdrew and those who had virologic failure. The least-squares mean difference also remained significant $(\mathrm{P}<0.05)$ in each of the cohort analyses performed at time points up to week 24. At week 24, the proportion of patients in each category of response was significantly greater in the enfuvirtide group than in the control group (Table 2).

The percentage of patients with virologic failure by week 8 was greater in the control group (33.3 percent) than in the enfuvirtide group (16.0 percent), and this difference persisted to week 24 (control group, 64.2 percent; enfuvirtide group, 41.7 percent). The distribution of the time to virologic failure was significantly different between the two groups ( $\mathrm{P}<0.001$ by the log-rank test) (Fig. 2). The median time to virologic failure was 99 days in the control group but could not be estimated in the enfuvirtide group.

\section{Changes in CD4+Cell Counts}

At week 24, the increase from base line in the CD4+ cell count was significantly greater in the enfuvirtide group than in the control group (Table 2).

\section{SAFETY}

\section{Injection-Site Reactions}

At week 24, nearly all patients in the enfuvirtide group ( 98.2 percent) had had at least one injectionsite reaction, with most having their first reaction during week 1 . Among patients who had pain or discomfort from injection-site reactions, most had either mild tenderness (49.7 percent) or moderate pain without limitation of usual activities (41.7 percent); 8.7 percent had pain or discomfort requiring nontopical analgesic agents or limiting usual activities, and none required hospitalization. Frequent symptoms of injection-site reactions included erythema (in 87.1 percent of patients), induration (in 84.0 percent), and nodules and cysts (in 81.6 percent). There was no evidence of an increase in the severity of injection-site reactions over time. Only small percentages of patients ( 2.8 percent in the enfuvirtide group and 1.2 percent in the group that switched to enfuvirtide) discontinued treatment with enfuvirtide because of injection-site reactions. 


\begin{tabular}{|c|c|c|c|c|c|}
\hline Variable & $\begin{array}{l}\text { Enfuvirtide } \\
\text { Group }\end{array}$ & $\begin{array}{l}\text { Control } \\
\text { Group }\end{array}$ & $\begin{array}{l}\text { Difference between } \\
\text { Groups }(95 \% \mathrm{Cl})\end{array}$ & $\begin{array}{l}\text { Odds Ratio } \\
\text { (95\% Cl) }\end{array}$ & $\begin{array}{c}P \\
\text { Value }\end{array}$ \\
\hline $\begin{array}{l}\text { Least-squares mean change from base line in plasma HIV-1 } \\
\text { RNA level }\left(\log _{10} \text { copies } / \mathrm{ml}\right) \dagger\end{array}$ & -1.696 & -0.764 & $0.933(0.594-1.271)$ & & $<0.001$ \\
\hline$<50$ Copies of HIV-1 RNA/ml of plasma (\% of patients) & 19.6 & 7.3 & & $3.30(1.70-6.39)$ & $<0.001$ \\
\hline$<400$ Copies of HIV-1 RNA/ml of plasma (\% of patients) & 31.7 & 16.4 & & $3.17(1.96-5.13)$ & $<0.001$ \\
\hline $\begin{array}{l}\text { Reduction from base line of } \geq 1 \log _{10} \text { copies of HIV-I RNA } \\
\text { per milliliter of plasma (\% of patients) }\end{array}$ & 51.8 & 29.1 & & $2.64(1.77-3.95)$ & $<0.001$ \\
\hline Least-squares mean increase in CD4+ cell count (cells $/ \mathrm{mm}^{3}$ ) & 76.2 & 32.1 & $44.1 \quad(22.5-65.8)$ & & $<0.001$ \\
\hline
\end{tabular}

* Plasma samples were obtained at every visit, and quantitative analysis of HIV-1 RNA levels was performed by a central laboratory (Covance Central Laboratory Services, Indianapolis) with the Amplicor HIV-1 Monitor, version 1.5 (Roche). Samples found by this test to contain fewer than 400 copies of HIV-1 RNA per milliliter were retested with ultrasensitive preparation of the sample in order to detect as few as 50 copies per milliliter. CD4+ cell counts were assessed with the use of standard techniques for flow cytometry. The last-observation-carried-forward method was used for the analysis of least-squares mean changes. $\mathrm{Cl}$ denotes confidence interval.

$\dagger$ A negative number represents a decrease.

\section{Adverse Events at Week 24}

Because of the 2:1 ratio for randomization, the design of the study allowing patients in the control group to switch to enfuvirtide, and the lower rate of virologic failure in the enfuvirtide group after 24 weeks of treatment, the total number of patientyears of exposure to the randomly assigned treatment regimen was approximately 2.5 times as high in the enfuvirtide group (162.8 patient-years) as in the control group (64.9 patient-years). After 24 weeks, 77.6 percent of patients in the enfuvirtide group and 74.5 percent in the control group had had an adverse event (excluding injection-site reactions) that was related to the treatment regimen. Diarrhea, nausea, and fatigue were the most frequently reported treatment-related adverse events in both groups (Table 3). Peripheral neuropathy and decreased appetite were the only treatment-related adverse events that occurred with a frequency at least 5 percent higher in the enfuvirtide group than in the control group.

Overall, 22 patients in the enfuvirtide group (6.7 percent) and 8 patients in the control group ( 4.8 percent) had adverse events with onset before week 24 that led to withdrawal from the study. The most frequent adverse events leading to withdrawal were vomiting in both groups (in 1.2 percent of patients in the enfuvirtide group and 1.2 percent of those in the control group), nausea in both groups (in 0.9 percent of patients in the enfuvirtide group and 1.2 percent of those in the control group), and diarrhea in the control group (1.2 percent). All other adverse

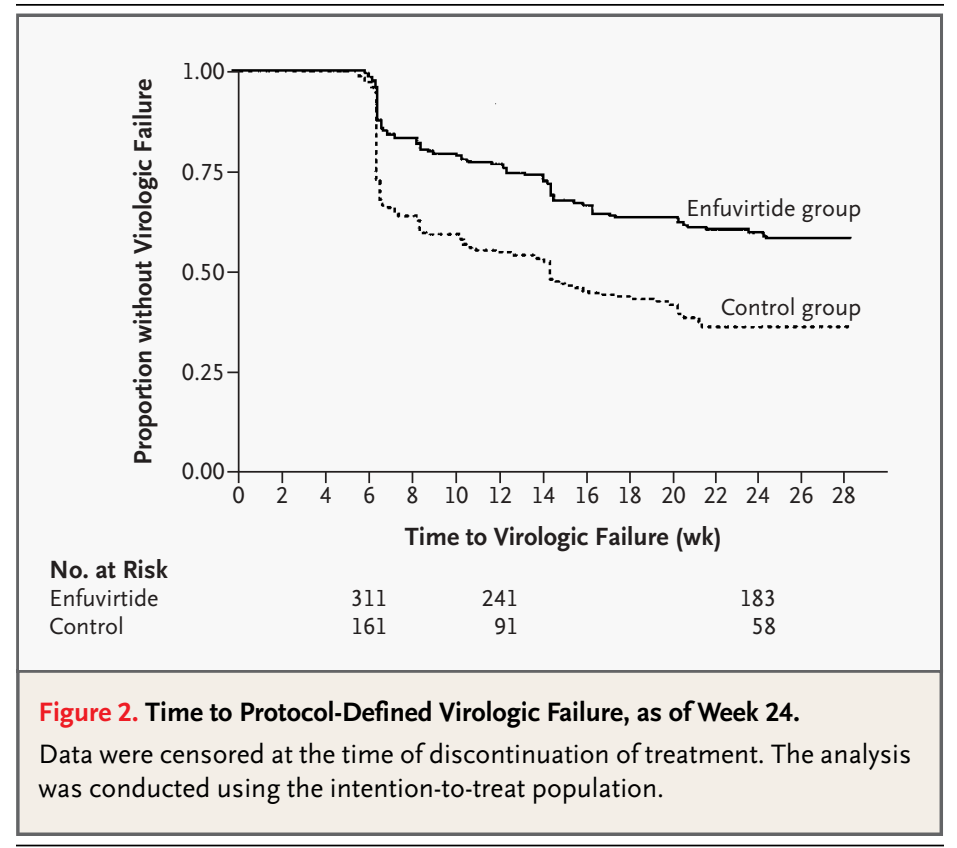

events leading to withdrawal occurred in 0.6 percent of patients or less. Two patients who switched to enfuvirtide (2.5 percent) had adverse events that began after the switch and subsequently led to withdrawal from the study; these events were diarrhea in one patient and progression of the acquired immunodeficiency syndrome in the other patient. Similar percentages of patients in the two groups died (1.2 percent [four patients] in the enfuvirtide group and 2.4 percent [four patients] in the control group) or 


\begin{tabular}{|lcc|}
\hline \hline $\begin{array}{l}\text { Table 3. Treatment-Related Adverse Events Occurring in at Least } 5 \text { Percent } \\
\text { of the Patients in Either Group. }\end{array}$ & $\begin{array}{c}\text { Enfuvirtide Group } \\
\text { (N=326) }\end{array}$ & $\begin{array}{c}\text { Control Group } \\
\text { (N=165) }\end{array}$ \\
\hline Variable & \multicolumn{1}{c}{ number (percent) } \\
Patients with $\geq 1$ event & $253(77.6)$ & $123(74.5)$ \\
Adverse event & $79(24.2)$ & $63(38.2)$ \\
Diarrhea* & $72(22.1)$ & $48(29.1)$ \\
Nausea & $64(19.6)$ & $28(17.0)$ \\
Fatigue & $36(11.0)$ & $9(5.5)$ \\
Peripheral neuropathy* & $32(9.8)$ & $10(6.1)$ \\
Insomnia & $29(8.9)$ & $15(9.1)$ \\
Headache & $26(8.0)$ & $5(3.0)$ \\
Decreased appetite* & $25(7.7)$ & $21(12.7)$ \\
Vomiting* & $24(7.4)$ & $7(4.2)$ \\
Dizziness (except vertigo) & $18(5.5)$ & $6(3.6)$ \\
Weight loss & $17(5.2)$ & $13(7.9)$ \\
Flatulence & & \\
\hline
\end{tabular}

* Data are for events that are not otherwise specified or not elsewhere classified.

had a serious adverse event while receiving the treatment to which they were originally assigned ( 25.8 percent in the enfuvirtide group and 21.2 percent in the control group).

\section{Updated Safety Analysis}

The updated safety analysis included 663 patients in the enfuvirtide groups and 334 patients in the control groups from TORO 1 and TORO 2. At the time of the updated analysis, 229 patients originally assigned to the control group had switched, adding enfuvirtide to their regimen. Because of the 2:1 ratio of randomization and the study design that allowed switching to enfuvirtide, patients in the original enfuvirtide group had 813 patient-years of exposure (median, 1.48 years per patient; range, $<0.01$ to 1.92) and patients in the control group had 163 patient-years of exposure (median, 0.35 year per patient; range, 0.04 to 1.60 ; ratio, 5:1). Patients exposed to enfuvirtide after switching had $214 \mathrm{pa}$ tient-years of exposure (median, 1.08 years per patient; range, $<0.01$ to 1.71 ). Results were adjusted for exposure (presented in terms of the number of patients with an event per 100 patient-years of exposure), with all the patients exposed to enfuvirtide combined into one group (with a total of 1027 patient-years of exposure) and compared with the control group.

This update generally confirmed the safety profile seen at 24 weeks, with the following observations. Although the overall incidence of bacterial infections was similar in the two treatment groups after adjustment for exposure (183 patients in the combined enfuvirtide groups [20.5 percent], or 17.8 per 100 patient-years of exposure, and 30 patients in the control group [ 9.0 percent], or 18.4 per 100 patient-years of exposure; $\mathrm{P}=0.56$ ), pneumonia, primarily bacterial, occurred more frequently in the combined enfuvirtide group (50 patients [5.6 percent], or 4.9 per 100 patient-years) than in the control group (1 patient [0.3 percent], or 0.6 per 100 patient-years; $\mathrm{P}=0.02$ ). Sepsis occurred more frequently in the combined enfuvirtide group (16 patients [1.8 percent], or 1.6 per 100 patient-years, vs. 2 patients [0.6 percent], or 1.2 per 100 patientyears, in the control group), but the exposure-adjusted rates were not significantly different $(\mathrm{P}=0.37)$.

Two patients had cases of systemic hypersensitivity reaction (both in TORO 1) that were considered to be related to enfuvirtide therapy, and both recurred on rechallenge. In the first patient, who was taking enfuvirtide in combination with didanosine, stavudine, amprenavir, and ritonavir, rash, fever, and vomiting developed after eight days of treatment. On rechallenge on days 17 and 22 of the study, rash, fever, and vomiting recurred within hours after the administration of enfuvirtide. The eosinophil count increased progressively from 280 per cubic millimeter at base line (upper limit of normal, 570 per cubic millimeter) to 350 per cubic millimeter on day 12 of the study and 690 per cubic millimeter on day 15 . Membranoproliferative glomerulonephritis developed in the second patient after 57 days of therapy with enfuvirtide in combination with tenofovir, lamivudine, lopinavirritonavir, amprenavir, and efavirenz. This patient had a history of diabetes, seasonal allergies, proteinuria, and hematuria. On rechallenge with all antiretroviral drugs on day 223, severe respiratory distress developed. No eosinophilia or increase from base line in the eosinophil count was noted.

Treatment-related eosinophilia ( $>700$ cells per cubic millimeter) occurred in a greater proportion of patients in the enfuvirtide group (74 of $662 \mathrm{pa}-$ tients who could be evaluated [11.2 percent], or 11.5 patients per 100 patient-years) than in the control group ( 8 of 332 patients who could be eval- 
uated [2.4 percent], or 4.9 patients per 100 patientyears). Eosinophilia was not associated with clinical events suggestive of systemic hypersensitivity.

Aside from eosinophilia, differences between the groups in the incidence of treatment-related grade 3 or grade 4 laboratory abnormalities were small, and no consistent pattern was evident to suggest a definitive association of enfuvirtide with any particular laboratory abnormality.

\section{DISCUSSION}

Our trial was designed specifically to evaluate a new class of anti-HIV-1 compounds in patients who have received treatment with multiple drugs. Resistance testing was used to construct an optimized background regimen for all patients in the trial. Along with the criteria for switching to enfuvirtide and the use of lopinavir-ritonavir and tenofovir, this approach allowed patients access to the best possible treatment options. This fact is reflected by the relatively high proportion of patients in the control group with responses to treatment despite the extensive resistance to antiretroviral drugs in this population of patients. This positive outcome was also due in part to the high level of adherence to treatment among patients in the control group $(\geq 85$ percent adherence in 90.3 percent of patients). The benefit of enfuvirtide was demonstrated by significant differences between the two treatment groups at week 24 in the magnitude of the reduction in the plasma HIV-1 RNA level, the proportion of patients in each category of virologic response, the distribution of time to virologic failure, and the increase in the CD4+ cell count.

Although the last-observation-carried-forward method can overestimate the individual drug effect if the HIV-1 RNA level rebounds quickly after virologic failure, it was chosen to provide a consistent rule for handling patients in either group who discontinued treatment or had virologic failure, as well as for those who switched to enfuvirtide. ${ }^{18,19}$ The robustness of the primary result was confirmed by three stringent sensitivity analyses that clearly showed that the magnitude of the estimate of the effect of enfuvirtide treatment was not determined primarily by the method of imputation.

Overall, except for local injection-site reactions, the safety and tolerability of enfuvirtide in combination with an optimized background regimen were similar to those of the background regimen alone over the course of 24 weeks of therapy. The safety results obtained from the combined TORO 1 and TORO 2 studies after longer exposure to enfuvirtide showed a higher rate of pneumonia among patients receiving enfuvirtide than among patients in the control group, but the overall incidence of bacterial infection was similar in the two groups.

Two patients had a hypersensitivity reaction that was considered to be related to enfuvirtide therapy and that recurred with rechallenge. There was a higher incidence of eosinophilia among patients receiving enfuvirtide, even after adjustment for the duration of exposure. Review of the cases of individual patients with eosinophilia did not reveal any clinical adverse events suggestive of hypersensitivity to enfuvirtide.

Injection-site reactions were the most common events associated with enfuvirtide treatment, occurring in most patients who received the drug, but pain or discomfort requiring analgesics or limiting usual activities occurred in only 8.7 percent. Only a small number of patients discontinued enfuvirtide therapy because of an injection-site reaction (2.8 percent of patients assigned to the enfuvirtide group and 1.2 percent of patients who switched to enfuvirtide). There was a high rate of adherence to enfuvirtide treatment, suggesting that injection-site reactions were not treatment-limiting.

In our study, enfuvirtide resulted in significant improvement in virologic and immunologic responses as compared with individualized, optimized, combination antiretroviral therapy alone. These findings are supported by the similar results obtained in the TORO 2 trial. ${ }^{15}$ These two studies provide firm proof of principle that HIV-1 glycoprotein 41 is a viable target for effective treatment of HIV-1 infection. A week-48 analysis will be performed in both trials to assess the durability of the response to enfuvirtide. The introduction of enfuvirtide as the first of this new class of antiretroviral agent could make an important contribution to the successful, individualized treatment of growing numbers of patients who have limited remaining treatment options.

Supported by Roche and Trimeris.

Dr. Lalezari reports having received consulting and lecture fees from Roche and Trimeris. Dr. Henry reports having received consulting or lecture fees from Abbott, Agouron, Bristol-Myers Squibb, Gilead, GlaxoSmithKline, and Merck and grant support from Bristol-Myers Squibb, GlaxoSmithKline, and Roche. Dr. Montaner reports having received consulting or lecture fees or grant support from Abbott, Agouron, Shire BioChem, Boehringer Ingelheim, BristolMyers Squibb, Dupont Pharma, Gilead Sciences, Glaxo Wellcome, Roche, Kucera Pharmaceutical, Merck Frosst, and Pharmacia \& Upjohn. Dr. Piliero reports having received consulting and lecture fees from Roche Laboratories and grant support from Roche and 
Trimeris. Dr. Trottier reports having received consulting or lecture fees from Roche, Bristol-Myers Squibb, Agouron, and Abbott. Dr Walmsley reports having received consulting or lecture fees from Abbott, Aguoron, Boehringer Ingelheim, Bristol-Myers Squibb, Dupont, GlaxoSmithKline, and Merck and grant support from Abbott, GlaxoSmithKline, and Boehringer Ingelheim. Dr. Cohen reports having received consulting fees, lecture fees, or gran support from Roche, Bristol-Myers Squibb, Abbott, Gilead, Boehringer Ingelheim, GlaxoSmithKline, and Trimeris. Dr. Ku- ritzkes reports having received consulting or lecture fees from Abbott, Bayer-Visible Genetics, Bristol-Myers Squibb, GileadTriangle, GlaxoSmithKline, Merck, Roche, Shire BioChem, Serono, Trimeris, Ortho Biotech, and ViroLogic and grant support from Roche, Trimeris, GlaxoSmithKline, Bristol-Myers Squibb, and Tanox. Dr. Eron reports having received consulting and lecture fees from Roche and grant support from Abbott, Merck, Roche, and Trimeris.

We are indebted to the patient volunteers who took part in the trial.

A P PEN DIX

In addition to the authors, the TORO 1 Study Group included the following institutions and persons: $\mathrm{C}$. Farthing and E. Graham (AHF Research, Los Angeles); M. Packard and L. Ngo (AIDS Clinical Research Unit, University of North Carolina at Chapel Hill, Chapel Hill); M. Lederman and J. Baum (AIDS Clinical Trials Unit, Case Western Reserve University, University Hospitals of Cleveland, Cleveland); R. Pollard, S. Rauf, and W. Silkowski (AIDS Clinical Trials Unit, University of Texas Medical Branch, Galveston); M. Thompson and A. Rucker (AIDS Research Consortium of Atlanta, Atlanta); M. Harris and G. Larsen (AIDS Research Program, St. Paul's Hospital, Vancouver, B.C., Canada); S. Preston and D. Cunningham (Albany Medical College, Albany, N.Y.); D. Guimaraes, A. Bertasso, and T. Kinchelow (Roche, Nutley, N.J.); R. Myers and B. Casimir (Body Positive, Phoenix, Ariz.); P.R. Skolnik and B. Adams (Boston University Medical Center, Boston); O.H.M. Leite and M. Oliveira (Casa da AIDS, Hospital das Clínicas da Faculdade de Medicina da Universidade de São Paulo, São Paulo, Brazil); E. Lefebvre and B. Gomez (Clinique Médicale l'Actuel, Montreal); K.B. Foy (Community Research Initiative of New England, Boston); H. Lampiris and S. Charles (Department of Veterans Affairs Medical Center, San Francisco, and University of California, San Francisco); J. Dobkin and M. Crawford (Division of Infectious Diseases, Columbia University, New York); T. Slom, R. Murphy, and T. Mikaitis (Division of Infectious Diseases, Northwestern University, Chicago); J. Witek and R. Anthony (Drexel University College of Medicine, Philadelphia); G. Richmond and V.F. Appleby (Fort Lauderdale, Fla.); F. Smaill and L. Kelleher (Hamilton Health Sciences, McMaster University Hospital, Hamilton, Ont., Canada); L. Nieto and S. Trevino (Hospital Gabriel Mancera, Mexico City, Mexico); M. Schechter and B. Fonseca (Hospital Universitario Clementino Fraga Filho, Universidade Federal do Rio de Janeiro, Rio de Janeiro, Brazil); E. DeJesus and R. Ortiz (Infectious Diseases Consultants Research Initiative, Altamonte Springs, Fla.); J. Wheat, M. Goldman, and D.K. O'Connor (Division of Infectious Diseases, Indiana University, Indianapolis); J.G. Sierra-Madero and S. Niño-Oberto (Instituto Nacional de Ciencias Médicas y Nutrición Salvador Zubirán, Mexico City, Mexico); J.E. Gallant and L. Apuzzo (Johns Hopkins University School of Medicine, Baltimore); N. Basgoz and K. Habeeb (Massachusetts General Hospital AIDS Clinical Trials Unit, Boston); P. Alpert and S. Thomas (Montefiore Medical Center, New York); T. Miller and T. Kempner (Oregon Health and Science University, Portland); P.R. Wolfe and J. Bautista (Pacific Oaks Research, Beverly Hills, Calif.); H.L. Martin and M.E. Morton (Park Nicollet Medical Center, St. Louis Park, Minn.); D. Henry and S. Kilcoyne (Pennsylvania Oncology Hematology Associates, Philadelphia); E. Glutzer (Quest Clinical Research, San Francisco); C. Rivera-Vazquez and Z. Pomales (Veterans Affairs Medical Center, San Juan, Puerto Rico); N. Bellos and L.A. Hoffman (Southwest Infectious Disease Associates, Dallas); B. Olmscheid, O. Klein, and M. Miller (St. Vincent's Medical Center, New York); C.R. Steinhart and A. Liebmann (Steinhart Medical Associates, Miami); S. Williams and L. Springate (The Vancouver Clinic, Vancouver, Wash.); K. Logue (Toronto Hospital, University of Toronto, Toronto); L. Smiley and G.D. Miralles (Trimeris, Durham, N.C.); R. Haubrich and K. Nuffer (University of California, San Diego, San Diego); G. Beatty and S. O'Leary (University of California, San Francisco, HIV Clinical Trials Group at San Francisco General Hospital, San Francisco); D. Rouleau and S. Dufresne (Unité Hospitalière de Recherche, d'Enseignement et de Soins sur le SIDA du Centre Hospitalier de l'Université de Montréal, Hôpital Notre-Dame, Montreal); J.M. Kilby, M. Saag, and K. Upton (University of Alabama at Birmingham, Birmingham); J. Feinberg and P. Kohler (University of Cincinnati, Cincinnati); T.B. Campbell and B.A. Putnam (University of Colorado Health Science Center, Denver); S.A. Riddler and R.R. Rosener (University of Pittsburgh, Pittsburgh); B.J. Barnett and I. Hansen (University of Texas Houston Medical School, Houston); A.C. Collier and B.A. Royer (University of Washington AIDS Clinical Trials, Seattle); D.W. Haas and M. Morgan (Vanderbilt AIDS Clinical Trials Center, Nashville); K. Sathasivam and J. Hersch (Whitman-Walker Clinic, Washington, D.C.).

REFERENCES

1. Hoetelmans RM. Sanctuary sites in HIV-1 infection. Antivir Ther 1998;3:Suppl 4:13-7.

2. Lafeuillade A, Solas C, Halfon $P$, Chadapaud S, Hittinger G, Lacarelle B. Differenc es in the detection of three HIV-1 protease inhibitors in non-blood compartments: clinical correlations. HIV Clin Trials 2002;3: 27-35.

3. Dieleman JP, Jambroes M, Gyssens IC et al. Determinants of recurrent toxicitydriven switches of highly active antiretroviral therapy: the ATHENA cohort. AIDS 2002;16: 737-45.

4. Moyle G, Carr A. HIV-associated lipodystrophy, metabolic complications, and antiretroviral toxicities. HIV Clin Trials 2002 3:89-98.

5. Dresser GK, Spence JD, Bailey DG. Pharmacokinetic-pharmacodynamic consequences and clinical relevance of cytochrome
P450 3A4 inhibition. Clin Pharmacokine 2000;38:41-57.

6. Casado JL, Sabido R, Perez-Elias MJ, et al. Percentage of adherence correlates with the risk of protease inhibitor treatment failure in HIV-infected patients. Antivir Ther 1999;4:157-61.

7. Raboud JM, Harris M, Rae S, Montaner JS. Impact of adherence on duration of virological suppression among patients receiving combination antiretroviral therapy. HIV Med 2002;3:118-24.

8. Race E, Dam E, Obry V, Paulous S, Clave F. Analysis of HIV cross-resistance to protease inhibitors using a rapid single-cycle recombinant virus assay for patients failing on combination therapies. AIDS 1999;13 2061-8.

9. Van Vaerenbergh $\mathrm{K}$, Van Laethem $\mathrm{K}$, Albert J, et al. Prevalence and characteristics of multinucleoside-resistant human immu- nodeficiency virus type 1 among European patients receiving combinations of nucleoside analogues. Antimicrob Agents Chemother 2000;44:2109-17.

10. Wild C, Greenwell T, Matthews T. A synthetic peptide from HIV-1 gp41 is a potent inhibitor of virus-mediated cell-cell fusion. AIDS Res Hum Retroviruses 1993;9:1051-3. 11. Kilby JM, Hopkins S, Venetta TM, et al. Potent suppression of HIV-1 replication in humans by T-20, a peptide inhibitor of gp41mediated virus entry. Nat Med 1998;4:1302-7. 12. Kilby JM, Lalezari JP, Eron JJ, et al. The safety, plasma pharmacokinetics, and antiviral activity of subcutaneous enfuvirtide (T-20), a peptide inhibitor of gp41-mediated virus fusion, in HIV-infected adults. AIDS Res Hum Retroviruses 2002;18:68593. [Erratum, AIDS Res Hum Retroviruses 2003;19:83.]

13. Lalezari JP, DeJesus E, Northfelt DW, et 
al. A controlled phase II trial assessing three doses of enfuvirtide (T-20) in combination with abacavir, amprenavir, ritonavir, and efavirenz in non-nucleoside reverse transcriptase inhibitor-naive HIV-infected adults. Antiviral Ther (in press).

14. Lalezari JP, Eron JJ, Carlson $M$, et al A phase II clinical study of the long-term safety and antiviral activity of enfuvirtidebased antiretroviral therapy. AIDS 2003;17 691-8.

15. Lazzarin A, Clotet B, Cooper D, et al.
Efficacy of enfuvirtide in patients infected Institute of Allergy and Infectious Diseases, with drug-resistant HIV-1 in Europe and 1996.

Australia. N Engl J Med 2003;348:2186-95. 18. Little R, Yau L. Intent-to-treat analysis 16. DeGruttola V, Dix L, D'Aquila R, et al. for longitudinal studies with drop-outs. BioThe relation between baseline HIV drug metrics 1996;52:1324-33.

resistance and response to antiretroviral ther- 19. Le Corfec E, Chevret S, Costagliola D. apy: re-analysis of retrospective and prospec- Visit-driven endpoints in randomized HIV/ tive studies using a standardized data analy- AIDS clinical trials: impact of missing data sis plan. Antivir Ther 2000;5:41-8.

17. AIDS Clinical Trials Group. Table of mary statistics. Stat Med 1999;18:1803grading severity of adult adverse experiences. 17.

Rockville, Md.: Division of AIDS, National Copyright $\odot 2003$ Massachusetts Medical Society. 